\title{
A European neutrino program based on the machine upgrades of the LHC
}

\section{A. Donini, E. Fernandez, S. Rigolin}

I.F.T. and Dep. Física Teórica, U.A.M., Madrid, Spain

\section{P. Migliozzi, L. Scotto Lavina}

Istituto Nazionale di Fisica Nucleare, Sez. di Napoli, Italy

\section{T. Tabarelli de Fatis}

Università di Milano-Bicocca and INFN, Milano, Italy

\section{F. Terranova*}

Laboratori Nazionali di Frascati dell'INFN, Frascati, Italy

E-mail: francesco.terranova@cern.ch

In the next decade, a significant upgrade of the LHC injection system is planned with the aim of fully exploiting the physics potential of this collider. In particular, the upgrade of the SPS (Super-SPS) would allow proton injection with energy twice the one of the current system and would ground on a firm basis the luminosity upgrade of the Large Hadron Collider. Beyond LHC physics, this machine offers an unprecedented opportunity for the experimental determination of the leptonic mixing matrix, particularly of Dirac CP violation. In this talk, we show that a Beta Beam based on the Super-SPS and on a 40 kton iron detector at Gran Sasso can address CP violation in the leptonic sector for any value of the $\theta_{13}$ angle that gives a positive $v_{\mu} \rightarrow v_{e}$ signal in the forthcoming SuperBeam experiments (e.g. T2K). In general, we show that a Beam Beam driven by the Super-SPS has a physics potential comparable to so-called "Phase II" SuperBeams (e.g. JAERI to HyperKamiokande) but does not require the construction of ultra-massive detectors and offers a fascinating synergy with high energy collider physics and nuclear physics.

International Europhysics Conference on High Energy Physics

July 21st - 27th 2005

Lisboa, Portugal

\footnotetext{
${ }^{*}$ Speaker.
} 


\section{Introduction}

In the next decades, long baseline experiments will provide access to the currently unknown entries of the leptonic mixing matrix (PMNS) if the mixing angle between the fi rst and third family $\left(\theta_{13}\right)$ turns out to be larger than $\simeq 1^{\circ}$. A large experimental effort is in progress to test the size of $\theta_{13}$ and in ten years from now (i.e. at the completion of the so called "Phase I" experiments like T2K, Nova, DoubleChooz) we expect to gain evidence for subdominant $v_{\mu} \rightarrow v_{e}$ transitions at the atmospheric scale as far as $\theta_{13} \lesssim 3^{\circ}$. In this case, however, new facilities will be needed to close up the PMNS. They should perform precision measurements of the 1-3 sector and particularly of the $\mathrm{CP}$ violating phase. Moreover, exploiting the perturbations of the oscillation probabilities occurring in matter, additional information concerning the neutrino hierarchy ( $\operatorname{sign}$ of $\Delta m_{32}^{2}$ ) could be obtained. A possible vision for these "Phase II" setups is based on the novel concept of Beta Beam and the strong potential synergy with the machine upgrades of the LHC planned beyond 2015, i.e. at a timescale comparable to the end of Phase I.

\section{The acceleration complex and the far detector}

A Beta Beam [1] is made by accelerating radioactive ions with a short beta-decay lifetime, by storing them in a ring with straight sections and by letting them decay. The focusing of the beam is provided by the Lorentz boost. Having the possibility to accelerate either $\beta^{-}$(e.g. ${ }^{6} \mathrm{He}$ ) or $\beta^{+}$(e.g. ${ }^{18} \mathrm{Ne}$ ) ions, pure $\bar{v}_{e}$ or pure $v_{e}$ beams can be produced, respectively. In particular, the Beta Beam concept offers a strong synergy with nuclear physics (high intensity radioactive beams) and low background contamination in the $v_{e} \rightarrow v_{\mu}$ channel. At present, the main drawback of this technique is related to the small energy of the parent ions. Working at $v$ energies of a few hundreds of $\mathrm{MeV}$ implies an enormous reduction of the cross section, complicates the kinematic reconstruction of the event and forces the choice of small baselines so that matter effects are suppressed. In this scenario, the physics performances of a Beta Beam equipped with a very large (1 Mton) water Cherenkov would be very similar to a multi-megawatt SuperBeam since the outstanding purity is compensated by the limited statistics. An increase of the Beta Beam energy [2] could be envisaged by a fast cycling superconducting SPS ("Super-SPS") at CERN. This machine is currently considered as an option for the luminosity upgrade and, possibly, the energy upgrade of the LHC [3]. In this case, the detector mass could be signifi cantly reduced and the baseline would match the CERNLNGS distance. In this confi guration, denser detectors operating in $v_{\mu}$ appearance mode can be exploited [4] and therefore smaller experimental halls are needed. Particularly, the existing halls of the Gran Sasso underground labs would be appropriate to host the far detector.

Working at high (above $1 \mathrm{GeV}$ ) neutrino energies opens the possibility to exploit iron calorimeters. On top of a good muon identifi cation, these detectors provide energy measurement of the hadronic shower produced in the neutrino interaction. The measurement of the muon momentum and of the hadronic shower allows for the reconstruction of the incident neutrino energy. The detector we considered for this study has been derived from a digital RPC based calorimeter proposed for the reconstruction of the energy flow at the ILC detector [5]. It consists of a sandwich of $4 \mathrm{~cm}$ non-magnetized iron and glass RPC. The active part of the RPC is segmented in $2 \times 2 \mathrm{~cm}^{2}$ elementary cells. A full Monte Carlo simulation has been carried out in order to evaluate the detector 
response, but the event classifi cation capability has only been based on inclusive variables (total number of hits and event length expressed in terms of number of crossed iron layers). The typical effi ciency for identifying a neutrino or anti-neutrino CC interaction is of the order of 50-60\%. The probability for the background to be identifi ed as a CC-like event is smaller than $1 \%$. A detailed description of the analysis can be found in [6].

\section{Sensitivity}

Since a detailed study of the expected fluxes from a Super-SPS based Beta beam is currently unavailable, we plot in Fig. 1 (left panel), for $\delta=90^{\circ}$, the minimum $\theta_{13}$ that can be distinguished from zero at $99 \%$ C.L. as a function of the flux ( 1 corresponds to $F_{0}$, i.e. the nominal fluxes assumed for the baseline design $[7,8]$ ). Notice that, if the flux is at least half of $F_{0}$, it is possible to discover a non vanishing $\theta_{13}$ even in the case of no signal observed in the T2K experiment. Assuming a flux equal to $F_{0}$, values of $\theta_{13}$ down to $1^{\circ}$ can be distinguished from zero. Fig. 1 (right panel) shows, for $\theta_{13}=3^{\circ}$, the minimum $\delta$ that can be distinguished from zero, at at $99 \%$ C.L., as a function of the neutrino flux. The value $\theta_{13}=3^{\circ}$ has been chosen being the minimum value for which T2K may discover a non-zero $\theta_{13}$. Also in this case, unless the flux is smaller than $F_{0} / 10$, it would be possible to establish CP violation in the leptonic sector for the whole $\theta_{13}$ range covered by T2K. A comparison with the baseline design for a Beta Beam at CERN can be found in $[6,9]$ together with the sensitivity in the occurrence of a null result $\left(\theta_{13}\right.$ limits as a function of $\delta$ ). Moreover, due to the large baseline and the capability of identifying spectral distortion of the $v_{\mu}$ signal, a Super-SPS based Beta Beam is able to distinguish normal from inverted neutrino mass hierarchies (sensitivity to the sign of $\Delta m_{31}^{2}$ ) for $\theta_{13} \gtrsim 6^{\circ}$ [6].
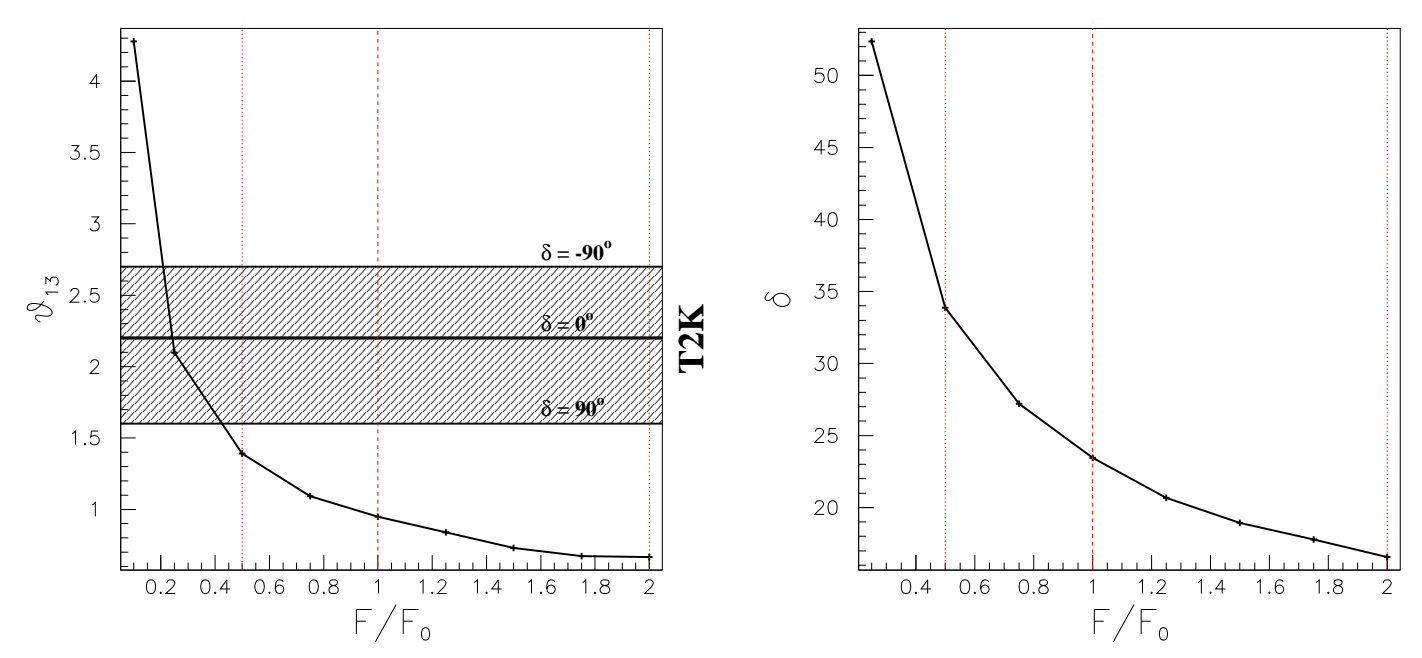

Figure 1: Left plot: minimum $\theta_{13}$ that can be distinguished from zero at $99 \%$ C.L. as a function of the flux ( 1 corresponds to $F_{0}$ ). Right plot: minimum $\delta$ that can be distinguished from zero, at $99 \%$ C.L., as a function of the neutrino flux. 


\section{Conclusions}

The Super-SPS option for the luminosity/energy upgrade of the LHC is optimal for the con-

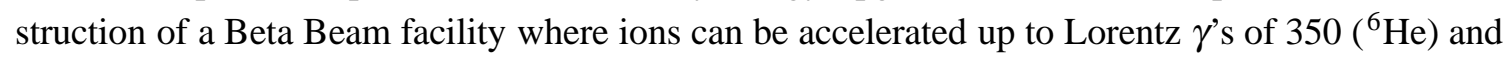
$580\left({ }^{18} \mathrm{Ne}\right)$. The physics case of this complex would be enormously strengthened in the case of $\theta_{13}$ discovery after the completion of the Phase I experiments $(\sim 2015)$. Since the upgrade of the injection complex of the LHC is foreseen after 2015, we see a window of opportunity for a Phase II neutrino program in Europe compatible with the LHC running. Moreover, the neutrino energy obtained exploiting the Super-SPS allows the use of very dense detectors (e.g. iron slabs interleaved with RPC) with a few tens of kiloton mass. This would fi $t$ into the existing underground facilities at Gran Sasso, whose distance from CERN happens to be at the peak of oscillation probability at the $\gamma$ 's mentioned above.

\section{References}

[1] P. Zucchelli, Phys. Lett. B 532 (2002) 166.

[2] J. Burguet-Castell et al., Nucl. Phys. B 695 (2004) 217.

[3] R. Garoby and W. Scandale, Nucl. Phys. Proc. Suppl. 147 (2005) 16; O. Bruning et al., CERN-LHC-PROJECT-REPORT-626, 2002; W. Scandale, High Intensity Injection Chain for LHC, talk at the High Intensity Frontier Workshop (HIF05), La Biodola, Italy, 2005.

[4] F. Terranova, Nucl. Phys. Proc. Suppl. 149 (2005) 185.

[5] A. Ghezzi, T. Tabarelli de Fatis, G. Tinti and M. Piccolo, arXiv:physics/0507021.

[6] A. Donini et al., A beta beam complex based on the machine upgrades of the LHC, in preparation.

[7] B. Autin et al., arXiv:physics/0306106. M. Benedikt, S. Hancock and M. Lindroos, in Proceedings of the 9th European Particle Accelerator Conference (EPAC 2004), EPAC-2004-MOPLT007.

[8] M. Mezzetto, arXiv:hep-ex/0511005, to appear in Nucl. Phys. B Proc. Suppl.

[9] A. Donini et al., arXiv:hep-ph/0511134. 